\title{
Experimental study and simplified mathematical description of preferential crystallisation
}

\author{
Martin Peter Elsner*,1), Dimas Fernández Menéndez ${ }^{2)}$ \\ Eva Alonso Muslera ${ }^{1)}$, Andreas Seidel-Morgenstern ${ }^{1,2)}$ \\ (1) Max Planck Institute for Dynamics of Complex Technical Systems, Sandtorstr. 1, 39106 Magdeburg, Germany
(2) Department for Chemical Engineering, Otto von Guericke University, Universitätsplatz 2, 39106 Magdeburg, Germany
}

\section{Abstract}

The purpose of this paper is to investigate the concept of preferential crystallisation focussing on aspects of quantification and application from the engineering point of view. In experiments performed, the amino acid threonine was used as a model system. Application of on-line polarimetry in combination with measurements of an on-line density meter proved the general applicability of this method in order to monitor directly the resolution progress, to recognise the region of "safe" resolution and to gain information about the crystallisation kinetics. For the evaluation of the crystal growth kinetics of the desired enantiomers the method of moments was applied to analyse isothermal single-step crystallisation experiments. A population balance model is used in order to simulate the time changes of liquid phase composition during the preferential crystallisation process. Finally, first experimental results regarding a cyclic operation mode will be presented.

Keywords: Enantioseparation, Entrainment effect, Conglomerates, Threonine, Method of moments, Crystal growth, Nucleation, Population balance

\section{Introduction}

The separation of chiral compounds is of large interest since most of the (bio-)organic molecules are chiral. Usually only one of the enantiomers shows the wanted properties with regard to therapeutic activities or metabolism, whereas the other enantiomer may be inactive or may even cause some undesired effects. For this reason enantiomeric separations have increasingly become important and their application ranges from the pharmaceutical and food industry to the agricultural industry. Generally, processes capable to gain enantiomers can be classified into the following groups: (1) classical non-biological resolutions via the formation of diastereomers, (2) biological methods (resolution and asymmetric synthesis), (3) non-biological asymmetric synthesis and (4) immobilisation and membrane technologies [1, 2].

An attractive alternative to these methods is enantioselective crystallisation exploiting the entrainment effect. This method is applicable for conglomerates. The principle of the pre-

\footnotetext{
* corresponding author

Phone: + 49-391-6110-438

Fax: + 49-391-6110-626

Email: mpelsner@mpi-magdeburg.mpg.de
} 
ferential crystallisation process can be illustrated in a ternary phase diagram. Figure 1 depicts the solvent and the two pure enantiomers in the corners of the diagram. Considering an initially undersaturated solution at a temperature $T_{\text {cryst }}+\Delta T$, the solution becomes supersaturated but remains clear, if it is rapidly cooled down to a temperature $T_{\text {cryst }}$ within the metastable zone. Retarded, its composition would change in order to reach the thermodynamic equilibrium. In the equilibrium state the liquid phase will have racemic composition (point E) and the solid phase will consist of a mixture of crystals of both enantiomers. However, after seeding with homochiral crystals it can be observed that the system does not reach the point $E$ directly but moves along other trajectories. Thus, under special conditions and in a restricted time interval it is possible to preferentially produce crystals of just one of the enantiomers. The functioning of this process is due to the homochiral surface area offered initially and the specific driving forces. Detailed treatises of the process of crystallisation by entrainment can be found in the literature, e.g. [3-5]. The potential of preferential crystallisation as an effective and alternative technology for the production of pure enantiomers has been the subject of considerable academic activities in the recent years with an emphasis on its chemistry [6] and on its application to separate special chiral systems [7]. Various experimental investigations have been published which, amongst other aspects, analyse the pre-treatment of the used seed crystals [8-10] or the influence of the size of the crystals on the transients of the purity drop [11].

The simplicity and potential of preferential crystallisation is also attractive for chemical engineers. From the engineering point of view existing processes have been usually developed pragmatically and not always following a "mass balanced based approach". This might be also a reason for the fact that this process is used only for a few substances in an industrial scale (like e.g. for $\alpha$-methyldopa [2, 12]).

\section{Single-step and cyclic operation of preferential crystallisation}

To further illustrate the process, Figure 2 presents typical transients of the liquid phase concentration which could be conventionally expressed for enantiomeric systems using the 
signal of a polarimeter [13]. The optical rotation angle of the mother liquor changes during the resolution process as a result of the composition change in the solution. In this figure it is assumed that the enantiomer $E_{1}$ reveals a negative specific rotation angle $[\alpha]_{\lambda}$, i.e (-)enantiomer. According to Figure 2, in case of an initial excess of enantiomer $E_{1}$ the curve starts with a negative value for the net optical rotation angle. After seeding with homochiral crystals, $E_{1}$ starts to crystallise and the solution becomes poor concerning this enantiomer. Consequently, the optical rotation angle increases until the nucleation of the counterenantiomer occurs. This region is not exactly defined and it is probably located near the maximum of the $\alpha$-signal. Up to this region reliable mathematical description is desirable for a meaningful process design. After that the second enantiomer $E_{2}$ increasingly crystallises and the measured optical rotation angle decreases up to a value of zero where the equilibrium state is reached. In order to obtain e.g. pure crystals of $E_{1}$ the batch process has to be stopped before reaching the maximum.

Since both enantiomers show the same properties concerning the crystal growth as well as nucleation kinetics, the examination of such a single-step should be sufficient for the estimation of kinetic parameters. The knowledge of the crystallisation kinetics is essential for the design as well as for controlling purposes of crystallisers. Furthermore, the physical description of the crystal growth and nucleation kinetics respectively, allows a prediction of several product features such as purity and crystal size distribution.

Regarding productivity a cyclic operation mode enables a "quasi-continuous" enantioseparation which is obviously much more attractive. The principle is illustrated schematically in Figure 3. An applicable configuration can consist of two crystallisers connected in series in which the separation of each enantiomer is carried out. The principle of this configuration is quite simple: In the upper vessel is initially a supersaturated solution of the racemate $\left(E_{1}+E_{2}\right)$. The presence of an initial enantiomeric excess in the first half cycle may have an influence on the resolution rate, but does not affect the general feasibility of the process. After addition of homochiral seeds, merely $E_{1}$ is crystallising within a limited time period. In order to gain this enantiomer as product of high purity, the process must be interrupted before the un- 
desirable counter-enantiomer occurs. For harvesting the pure solid product a filtration device is located between the crystallisation vessels in each case. After removing the product, the solution is located in the second vessel. To guarantee a quasi-continuous enantioseparation process a certain amount of racemate has to be added in advance and afterwards seeding with $E_{2}$ crystals will entail the crystallisation of $E_{2}$ in this following half cycle.

The goal of our work is to study experimentally the preferential crystallisation process for a model system. The amino acid L-/D-threonine in water was chosen as a suitable test substance. A single-step and a cyclic operation mode were investigated. The first one allows an estimation of kinetic data which provide together with an adequate model a basis for the mathematical description, whereas the latter demonstrates the possible quasi-continuous application of the process. The investigation is intended to shed light on the potential of the preferential crystallisation process and to provide first experience in bridging the gap between experimental results and theoretical concepts.

\section{Theoretical aspects}

\subsection{Modelling and simulation}

A perfectly mixed batch crystalliser can be simulated in a simplified manner using a dynamic, one-dimensional model assuming isothermal conditions and size-independent overall growth rates $\mathrm{G}^{(\mathrm{k})}$ and nucleation rates $\mathrm{B}^{(\mathrm{k})}$ for each of the two components $\mathrm{k}(\mathrm{L}-$, D-threonine). Below, these rates were considered to depend on the particle environment and not on the particle dimensions. This results in the following population balance [14]:

$$
\frac{\partial F_{N}^{(k)}(x, t)}{\partial t}=-\frac{\partial}{\partial x}\left(G^{(k)} \cdot F_{N}^{(k)}(x, t)\right)+B^{(k)} \cdot F_{\text {nuc }}^{(k)}(x, t), \quad k=L-, \text { D-threonine }
$$

with

$$
\mathrm{G}^{(\mathrm{k})}=\frac{\mathrm{dx}}{\mathrm{dt}} \text { and } \quad \mathrm{B}^{(\mathrm{k})}=\frac{\mathrm{dN}_{(\mathrm{k})}}{\mathrm{dt}} \text {. }
$$


The population of crystals is described by the number density functions $\mathrm{F}_{\mathrm{N}}$. The characteristic particle size is represented by $x$. The mass balance for the solution (continuous phase) is given by Eq. (2)

$$
\frac{d m_{(\text {liq })}^{(k)}}{d t}=-3 \rho_{s} k_{v} \cdot G^{(k)} \int_{0}^{\infty} x^{2} \cdot F_{N}^{(k)}(x, t) d x-\rho_{s} k_{v} \cdot B^{(k)} \int_{0}^{\infty} x^{3} \cdot F_{\text {nuc }}^{(k)}(x, t) d x
$$

For the solution the following boundary and initial conditions were applied:

$$
\begin{aligned}
& \text { Boundary condition: } F_{N}^{(k)}(0, t)=0 \\
& \text { Initial condition: } \quad F_{N}^{(k)}(x, 0)=F_{N, \text { seeds }}^{(k)}(x)
\end{aligned}
$$

The number population density functions $\mathrm{F}_{\mathrm{N}, \mathrm{seeds}}^{(\mathrm{k})}(\mathrm{x})$ of the invested seed crystals at the beginning of each experiment can be determined e.g. by microscopy. The population distributions $F_{\text {nuc }}^{(k)}$ of the nuclei are typically approximated by a DIRAC's function $\delta\left(x-x^{*}\right)$ with neglecting $x^{*} \approx 0$.

These balances represent a one-dimensional system of integro-partial differential equations, which were discretised by means of finite differences on a fixed CARTESIAN grid and then solved numerically over the temporal domain by MATLAB ${ }^{\circledR}$. An upwind discretisation scheme for the space coordinate was applied. It has been found that 4000 grid points in space were sufficient in order to minimise numerical dispersion and to achieve a satisfactory accuracy. The deficit in the total mass balances was less than $0.15 \%$. For the time scale the implemented "ODE23"-solver was applied which is based on an explicit RUNGE-KUTTA $(2,3)$ pair according to BOGACKI \& SHAMPINE [15] and which is in particular appropriate to solve moderately stiff problems.

Phenomena like agglomeration, abrasion and breakage were neglected in this first mathematical approach. More sophisticated models should take these phenomena into account in future. 


\subsection{Kinetic parameters and method of moments}

The common methods for the determination of crystal growth parameters in the field of crystallisation technology are subdivided into indirect and direct methods. The first one is based on the temporal measurement of the concentration in the mother liquor $[16,17]$, whereas the direct method is based on the temporal development of the size and/or mass of seed crystals [18].

In the present work, the method of moments is used which is a well-known method capable to simplify the complex evolution of property distributions. Detailed surveys about this method in relation to particulate processes are given in the literature [14, 19-22]. This approach could be applied to analyse the first period of an experiment before nucleation of the second enantiomer. Furthermore, assuming again a DIRAC distribution for the formed nuclei as mentioned above the temporal derivation of the $\mathrm{j}$-th moments can be summarised as follows:

$$
\begin{aligned}
& \frac{d \mu_{j}^{(k)}}{d t}=j \cdot G^{(k)} \cdot \mu_{j-1}^{(k)} \quad \text { for } j \geq 1 \\
& \frac{d \mu_{0}^{(k)}}{d t}=B^{(k)}
\end{aligned}
$$

where the $\mathrm{j}$-th moment is defined as

$$
\mu_{j}^{(k)}=\int_{0}^{\infty} x^{j} \cdot F_{N}^{(k)}(x) d x
$$

Using the general definition of moments, in particular, we obtain for the development of the third moment

$$
\frac{\mathrm{d} \mu_{3}^{(\mathrm{k})}}{\mathrm{dt}}(\mathrm{t})=3 \cdot \mathrm{G}^{(\mathrm{k})} \cdot \mu_{2}^{(\mathrm{k})}(\mathrm{t}) \sim \frac{\mathrm{d} \mathrm{V}_{\mathrm{s}}^{(\mathrm{k})}}{\mathrm{dt}}(\mathrm{t})=\frac{\mathrm{dV} \mathrm{V}_{\mathrm{s}}^{(\mathrm{k})}}{\mathrm{dt}}\left(\mathrm{w}_{1}(\mathrm{t}), \mathrm{w}_{2}(\mathrm{t}), \mathrm{c}_{\text {tot }}(\mathrm{t})\right)
$$

which is proportional to the volume changes of the solid phase depending on weight fractions $\mathrm{w}_{(\mathrm{k})}$ of both enantiomers as well as on the total concentration $\mathrm{c}_{\text {tot }}$ in the liquid phase. 
To estimate the development of selected moments on-line polarimetry was recently successfully used for monitoring the preferential crystallisation process by measuring the optical rotation angle of the solution and thereby, the difference of the enantiomers' mass concentrations $[23,24]$. The combination of on-line polarimetry with on-line density measurements enables to follow the progress of the concentrations of both, enantiomers in the mother liquor and the progress of the crystallised amount of enantiomers.

Furthermore, the analytical measurements of the composition in the mother liquor provide directly the temporal development of the solid mass of the crystallised species and therefore the third moment as it is shown below. The geometry of the formed crystals does not play any role for the determination of the third moment.

In performing experiments the difference between the weight fractions, $w_{D}$ and $w_{L}$ of both enantiomers in the solution can be measured polarimetrically

$$
\alpha_{m}(t)=k_{p o l} \cdot\left(w_{D}(t)-w_{L}(t)\right),
$$

whereas the total concentration can be determined from the experimentally accessible liquid phase density $\rho_{\text {sol }}$ :

$$
\rho_{\text {sol }}(t)=k_{d} \cdot\left(w_{D}(t)+w_{L}(t)\right)+\rho_{0} \text {. }
$$

A combination of both equations allows expressing the weight fraction changes as a function of the transients of the polarimetric signal and the solution density

$$
\begin{aligned}
& w_{D}(t)=\frac{m_{D}(t)}{m_{D}(t)+m_{L}(t)+m_{w}}=\frac{1}{2} \cdot\left(\frac{\rho_{\text {sol }}(t)-\rho_{0}}{k_{d}}+\frac{\alpha_{m}(t)}{k_{p o l}}\right), \\
& w_{L}(t)=\frac{m_{L}(t)}{m_{D}(t)+m_{L}(t)+m_{w}}=\frac{1}{2} \cdot\left(\frac{\rho_{\text {sol }}(t)-\rho_{0}}{k_{d}}-\frac{\alpha_{m}(t)}{k_{p o l}}\right) .
\end{aligned}
$$

In the case of a simultaneous crystallisation of both enantiomers, these two complementary information as well as their temporal derivatives can be taken for the calculation of the derivation of the third moment. For example the change of the third moment of L-threonine can be calculated using Eq. (13) 


$$
\frac{d \mu_{3}^{(1)}(t)}{d t}=\frac{\left(\frac{1}{k_{\text {pol }}} \cdot \frac{d \alpha_{m}}{d t}-\frac{1}{k_{d}} \cdot \frac{d \rho_{\text {sol }}}{d t}\right) \cdot\left(1-\frac{\rho_{\text {sol }}(t)-\rho_{0}}{k_{d}}\right)-\left(\frac{\rho_{\text {sol }}(t)-\rho_{0}}{k_{d}}-\frac{\alpha_{m}(t)}{k_{p o l}}\right) \cdot \frac{1}{k_{d}} \cdot \frac{d \rho_{s o l}}{d t}}{\frac{2 \rho_{s}}{m_{w}} \cdot\left(1-\frac{\rho_{s o l}(t)-\rho_{0}}{k_{d}}\right)^{2}} .
$$

For the exclusive crystallisation of L-threonine, this means that the counter-enantiomer remains in solution and its mass in solution is constant $\left(m_{D}=m_{D, 0}\right)$, the application of only one of the regarded analytical techniques is sufficient in order to determine the derivate of the third moment. Using the typically more precise polarimeter, it is possible to calculate the derivative of the third moment according to

$$
\frac{\mathrm{d} \mu_{3}^{(1)}(\mathrm{t})}{\mathrm{dt}}=\frac{1}{\rho_{\mathrm{S}}} \cdot \frac{2 \mathrm{~m}_{\mathrm{D}, 0}+\mathrm{m}_{\mathrm{w}}}{\left(1+\frac{\alpha_{\mathrm{m}}(\mathrm{t})}{\mathrm{k}_{\mathrm{pol}}}\right)^{2}} \cdot \frac{1}{\mathrm{k}_{\mathrm{pol}}} \cdot \frac{\mathrm{d} \alpha_{\mathrm{m}}}{\mathrm{dt}} .
$$

Thus, the development of the third moment is easily accessible based on the results of standard detectors.

In contrast, the transient of the second moment $\mu_{2}(t)$, corresponding to the total surface area depends on the geometry of the crystals and it is not possible to derive any information about its development by analysing only liquid phase properties. For this reason an independent experimental determination of the total surface area is indispensable. It can be realised for example by microscopy. Assuming e.g. an ideally cylindrical-shaped crystal the surface area $a_{i}$ can be evaluated by measuring the length $L_{C, i}$ and the width $D_{C, i}$ of each crystal i according to Eq. (15)

$$
a_{i}^{(k)}=\frac{\pi}{2} \cdot D_{C, i}^{(k) 2} \cdot\left(1+2 \cdot \frac{L_{C, i}^{(k)}}{D_{C, i}^{(k)}}\right) .
$$

In order to characterise a representative sample, this leads to an area distribution function $F_{A}$ which provides a mean value for surface area of a single crystal

$$
\overline{\mathrm{a}}^{(\mathrm{k})}=\int_{0}^{\infty} \mathrm{a}^{(\mathrm{k})} \mathrm{F}_{\mathrm{A}}\left(\mathrm{a}^{(\mathrm{k})}\right) d \mathrm{a}^{(\mathrm{k})}
$$


or in discretised form

$$
\overline{\mathrm{a}}^{(\mathrm{k})}=\sum_{(\mathrm{i})} \mathrm{a}_{\mathrm{i}}^{(\mathrm{k})} \mathrm{F}_{\mathrm{A}}^{(\mathrm{k})}\left(\mathrm{a}_{\mathrm{i}}^{(\mathrm{k})}\right) \Delta \mathrm{a}_{\mathrm{i}}^{(\mathrm{k})}
$$

which finally leads with the number of crystals $N_{\text {total }}$ to the total surface area of the "population" corresponding to the second moment:

$$
\mu_{2}^{(\mathrm{k})}=\overline{\mathrm{A}}_{\mathrm{total}}^{(\mathrm{k})}=\mathrm{N}_{\mathrm{total}}^{(\mathrm{k})} \cdot \overline{\mathrm{a}}^{(\mathrm{k})}
$$

$\mathrm{d} \mu_{3} / \mathrm{dt}$ and $\mu_{2}$ lead to the crystal growth rate $\mathrm{G}$ (Eq. 8) which describes the one-dimensional evolution of the effective particle diameter $x$. The effective particle diameter $x_{i}$ of a single, cylindrically shaped crystal can be calculated by Eq. (19)

$$
V_{i}^{(k)}=V_{i, s p h e r e}^{(k)} \Rightarrow x_{i}^{(k)}=\sqrt[3]{\frac{3}{2} \cdot D_{C, i}^{(k) 2} \cdot L_{C, i}^{(k)}}
$$

assuming that the actual volume of a single particle corresponds to the volume of a sphere with the diameter $x_{i}$.

In addition, the knowledge of this growth rate together with the second moment enables, after numerical determination of the temporal derivations of the remaining moments, the estimation of the nucleation rate B according to Eqs. (5-6).

\subsection{Crystal growth and nucleation rate models}

There is a variety of mechanisms of crystal growth, which might take place in competition and lead to transients [21]. Although more detailed models cited in the literature give a good insight into the mechanisms of crystal growth, they imply many parameters which are difficult to determine or predict. For this reason below a simple power law equation was chosen to quantify crystal growth

$$
\mathrm{G}^{(\mathrm{k})}=\mathrm{k}_{\mathrm{g}} \cdot\left(\mathrm{S}_{(\mathrm{k})}-1\right)^{\mathrm{g}}
$$

in which $\mathrm{S}_{(\mathrm{k})}$ denotes the actual degree of supersaturation for enantiomer $\mathrm{k}$ 


$$
S_{(k)}=\frac{W_{(k)}}{W_{e q,(k)}}
$$

and $\mathrm{k}_{\mathrm{g}}$ is an overall crystal growth rate constant.

It appears to be reasonable to assume that both enantiomers ( $k=1$ or 2$)$ obey the same crystal growth law. Furthermore, it is supposed that there is no interdependence of the growth rate for each of both enantiomers, i.e. that the growth function for the enantiomers $\mathrm{k}=$ 1 does not depend on the supersaturation of the counter-enantiomer $G^{(1)} \neq f\left(S_{(2)}\right)$.

If crystals are already dispersed in the crystallising medium, secondary nucleation can occur at supersaturation levels which are significantly lower than those at which primary nucleation takes place [21]. Phenomena like micro-breakage and abrasion might result in the formation of secondary nuclei. Based on previous experiments published in $[23,24]$ it is highly probable that for the desired (seeded) enantiomer secondary nucleation seems to be predominant. This can be described by

$$
B^{(1)}=k_{b}^{(1)} \cdot\left(S_{(1)}-1\right)^{b}
$$

where $k_{b}$ is generally assumed to be related to the stirring power. The correlation $B \sim G^{2}$ is often used as an approximation where the nucleation rate is proportional to the square of the growth rate [26].

For the case that the process is not stopped before reaching the maximum of the polarimetric signal, primary (heterogeneous) nucleation is assumed to initiate the crystallisation of the counter-enantiomer. To quantify this effect often the following semi-empirical expression can be used [21]

$$
B^{(2)}=k_{b}^{(2)} \cdot e^{-\frac{a(2)}{\ln ^{2} S_{(2)}}} .
$$

For the binary system of concern, the process development is illustrated in Figure 4. After seeding e.g. with L-threonine crystals at $t_{0}$, the crystallisation process is initiated immediately: The added seeds which are characterised by a number distribution function are growing and moreover, a formation of L-threonine nuclei is considered. Besides, a sudden nuclei "shower" 
of the counter-enantiomer is assumed to appear later at $t_{2}$. Of course, both kinds of nuclei start to grow after their formation.

\section{Materials and experimental set-up}

\subsection{Materials}

As mentioned already above the chiral substance used in the experimental studies was threonine (L-threonine: Merck, purity > 99\%; DL-threonine, D-threonine: Aldrich, purity = $98 \%$. As a basis the ternary solubility phase diagrams of threonine in water and waterethanol mixtures are known. They were determined in a previous study [26] using the classical isothermal method in an equilibrium apparatus.

\subsection{Preliminary experiments for preferential crystallisation}

A series of 26 preliminary experiments carried out for the investigation of the batchwise preferential crystallisation are summarised in Tab. 1. They serve to study effects of stirrer speed, seed amount, surface area of seeds, initial enantiomeric excess, extent of subcooling and crystallisation temperature. These studies were performed in similar experimental setups and with similar equipment as well as under comparable conditions mentioned below. Possible deviations can be found in this enumeration. Each run was repeated several times to check reproducibility. Most runs were performed until the equilibrium state was established. Further details are found in [23, 24].

\subsection{Single-step preferential crystallisation and growth kinetics}

A series of 11 single-step measurements for determining crystal growth kinetics was performed under identical conditions in a stirred and double-jacketed glass-made vessel of $1200 \mathrm{ml}$ total volume (Tab. 2: run I). At that time only one experimental data set with a subcooling of $\Delta \mathrm{T}=7 \mathrm{~K}$ and a seed mass of $2500 \mathrm{~g}$ was studied systematically for the crystal 
growth measurements. Further investigations will follow.

The temperature was controlled by a Pt-100 resistance thermometer (resolution $0.01 \mathrm{~K}$, uncertainty $1 / 3$ DIN). Crystal-free solution was pumped out of the crystalliser with a flow rate of $3.7 \mathrm{ml} / \mathrm{min}$ by a circulation pump (Heidolph PD 5201, SP Quick 1.6). The liquid phase was then separated using a sintered glass frit (pore width $0.45 \mu \mathrm{m}$ ) to prevent crystal removal. To avoid limitations due to mass transfer resistances and to guarantee reproducibility the experiments were performed with a constant stirring speed of $500 \mathrm{rpm}$ using a propeller stirrer. The solution was pumped in a thermostatted line along the way to the detector of the polarimeter (POLARmonitor, IBZ Messtechnik; length of cuvette: $60 \mathrm{~mm}$ ) and the density meter (DE 40, Mettler Toledo). Behind the analytical device the solution was recycled to the vessel by an insulated line. The measuring cells of the polarimeter as well as of the density meter were maintained at a constant temperature of $40^{\circ} \mathrm{C}$ to avoid recrystallisation. For the preferential crystallisation experiments the initial solutions were saturated at a temperature of $40^{\circ} \mathrm{C}$ according to the solubility data and specified enantiomeric excess. The solutions were heated up and maintained at a higher temperature for each case to ensure a complete dissolution of all crystals. Then the solution was cooled down to the desired crystallisation temperature. Afterwards, seed crystals of the enantiomer were provided in order to offer surface and to activate the resolution process. Even though the affect of pre-treatment like washing of the seed crystals was reported previously [12], in these experiments the seed crystals were taken without any treatment. Seed crystals of a sieve fraction between 212 and $300 \mu \mathrm{m}$ (concerning width D, cf. Figure 5) were used to avoid blocking of the sampling filter.

For the determination of the evolution of the surface area $A_{\text {total }}(t)$ and therefore $\mu_{2}(t)$, representative solid samples were after separation by filtration and washing with $5-9 \mathrm{ml}$ ice water (depending on the yield of the gained product) analysed by microscopy (Stemi 2000-C, Zeiss) every $30 \mathrm{~min}$ up to a total process time of $300 \mathrm{~min}$. For each sample at least 500 crystals showing an approximately cylindrical shape (compare Figure 5) were investigated and the surface area was determined by measuring the length $L$ and the width $D$ of each crystal. Since the evaluation of the surface area distribution functions is very time consuming, 
the experimental data set (cf. run I in Tab. 2) was fitted to obtain values for the whole investigated time scale of concern. In order to avoid strong disturbances of the process progress observed in distorted polarimetrical profiles described in [23], each experiment had to be repeated under the same conditions and stopped at different time. Moreover, these solid samples were analysed by chiral HPLC (column: Chiribiotic T, Astec, $5 \mu \mathrm{m}, 150 \times 4.6 \mathrm{~mm}$ ). HPLC analysis verified a product purity of $100 \%$ until a process time of $300 \mathrm{~min}$.

\subsection{Studies in cyclic operation mode}

A separation experiment in a cyclic operation mode according to the scheme shown in Figure 3 was performed (run II in Tab. 2) in a plant constructed for this purpose (see Figure 6). This facility consists of two crystallisers in series with (removable) filtration units between them for gaining the solid product. The double-jacketed glass-made filtration units of $250 \mathrm{ml}$ total volume were maintained at crystallisation temperature to avoid a further crystallisation by cooling during the filtration process. A frit (porosity P2) is located inside the filtration device. Accelerating of the filtration process was realised under vacuum. In order to prevent undesirable crystallisation outside the vessels, the whole periphery, i.e. all lines and the filtration devices, are thermostatted. In principle, the equipment and analytical facilities are comparable with those mentioned above. The process was performed for two subsequent separation cycles at a subcooling of $10 \mathrm{~K}$ and racemic composition at the beginning of the first cycle $\left(e_{0}=0 \%\right)$. These conditions which differ slightly from those for the crystal growth studies were deliberately chosen in order to check if the results based on the simplified mathematical model can also be extrapolated. For seeding $2500 \mathrm{mg}$ seed crystals of L- or Dthreonine (sieve fraction: 212-300 $\mu \mathrm{m}$ ) were used. To interrupt the crystallisation process a "stop" rotation angle $\alpha_{\text {stop }}$ was chosen corresponding to an enantiomeric excess of $2 \%$ of the counter-enantiomer. When the polarimetric signal achieved this value, the process was stopped and the solution was filtered. After addition and dissolution of fresh racemate as well as after seeding the crystallisation of the counter-enantiomer was performed. 


\section{Results \& discussion}

\subsection{Preliminary experiments: general studies on preferential crystallisation}

In preliminary investigations the influence of stirrer speed, seed amount, surface area of the seeds, initial enantiomeric excess, extent of subcooling, and crystallisation temperature has been investigated systematically. Moreover, experiments for the examination of the metastable zone width, reproducibility, process symmetry and scale-up behaviour have been also performed. Primary, the experiments were performed with different stirrer speeds. However, no reproducibility could be achieved at low stirrer speed of $200 \mathrm{rpm}$. It seems that the crystallisation process was strongly limited by mass transfer resistances. Thus, the stirring speed was increased up to $500 \mathrm{rpm}$ leading to a very good reproducibility. Furthermore, it could be shown that the amount as well as the offered surface area of seeds affects significantly the crystallisation rate and increasing the enantiomeric excess accelerates the process. The higher the supersaturation, the higher the yield and the faster both enantiomers crystallise. With regard to the alternative crystallisation of L- and D-threonine the expected symmetry of the optical rotation angle profiles and of the evolution of the mother liquor composition could be observed. Therefore, the investigation of one enantiomer seems to be sufficient for obtaining kinetic information. More details are given in [23, 24].

\subsection{Studies on the crystal growth kinetics and comparison with simulation results}

Results of a typical experiment (Tab. 2: run I), which was carried out for preferentially crystallising L-threonine at first, is shown in Figure 7. It shows the profiles of the optical rotation angle without an initial enantiomeric excess (left-hand) and the evolution of the mother liquor composition shown in a quasi-binary phase diagram (right-hand) until the equilibrium state was established. The evolution of the liquid phase composition represents the crystallisation pathway, starting without an initial enantiomeric excess, moving along a straight line and achieving a maximum enantiomeric excess of the counter-enantiomer D-threonine at the turning point. Then the solution tends to reach the equilibrium state (racemic composition at 
crystallisation temperature $T_{\text {cryst }}=33^{\circ} \mathrm{C}$ ) by degradation of the D-excess. Before the pathway changes its direction, only L-threonine is obtained in the solid phase whereas behind the turning point D-threonine simultaneously crystallises.

In the present work, the determination of the particle area distribution functions at several times provides the evolution of the mean values for the total surface area, i.e. the second moment (Eq. 18). As an example the distribution function $\mathrm{F}_{\mathrm{A}}$ of the particle surface area of the seed crystals (i.e. $t=0$ ) is shown in Figure 8 (the maximum errors are indicated).

The temporal development of the second moment as well as a desupersaturation curve is shown in the left-hand diagram of Figure 9. In addition, to enable the calculation of the crystal growth function $\mathrm{G}^{(1)}$ for L-threonine, the development of the third moment was determined by means of the changing polarimetric signal and the density of the solution according to Eq. (14). Using Eq. (8) the crystal growth rate $\mathrm{G}^{(1)}$ could be calculated for each experiment. Plots of the latter versus the respective supersaturation provide the information concerning the crystal growth kinetics. In the right-hand diagram of Figure 9 such a typical plot is exemplified for an experiment performed until $t=300 \mathrm{~min}$. A linear relationship between the crystal growth rate and the supersaturation seems to be a good approximation. The evaluation of the whole series of measurements leads to an averaged growth rate constant $\mathrm{k}_{\mathrm{g}}{ }^{(1)}$ of $(2.5 \pm 1.9) \cdot 10^{-7} \mathrm{~m} / \mathrm{s}$ and a first-order kinetics with respect to the relative supersaturation $S^{(1)}-1$ of L-threonine with $g=1.0 \pm 0.3$ under the prevalent operating conditions. Here it is emphasised that these values represent a first estimation at the present time. Further experiments under different conditions are currently investigated.

Obviously, the application of the method of moments does not seem to be suitable for an estimation of the nucleation rate $B$ if an off-line technique is used for the particle size characterisation. The main reason is the lack of a sufficient number of values for the development of the second moment using such a time consuming off-line method. The consecutive numerical iterations for the derivatives of the single moments corresponding to Eqs. (5-6) lead to a strong increase of the experimental errors. Measurements with a higher time resolution, realised e.g. by a (reliable) in-line technique, could reduce these numerical inaccu- 
racies. Besides, the size resolution of the applied microscope does not allow determining particles which are smaller than $100 \mu \mathrm{m}$. Thereby, an examination of small particles originating from nucleation seems to be very complicated. For this reason an estimation of the nucleation rate $B$ by the method of moments was not pursued.

However, using the estimated parameters for the crystal growth rate, the profiles for the optical rotation angle were simulated taking the exclusive crystallisation of L-threonine into consideration. Theoretical and experimental $\alpha$-curves are compared in Figure 10. Considering only the indicated time interval up to 300 min used for parameter estimation, the neglecting of nucleation $\left(\mathrm{k}_{\mathrm{b}}=\mathrm{O} \mathrm{s}^{-1}\right)$ results in an underestimation of the optical rotation angle. A better agreement is achieved between predicted and measured profiles within this time region if the nucleation is taken into account. As can be concluded from the results presented in Figure 10, the nucleation of the seeded enantiomer plays obviously an important role during the crystallisation process and has a pronounced effect on the course of the optical rotation angle in such transient experiments. Due to the correlation $B \sim G^{2}$ (see above), a quadratic approach with regard to the relative supersaturation was used. Secondary nucleation is usually considered to be promoted by the suspension density of which changes are slight in our experiments so that the dependence of the mass of crystallised solid per unit volume of suspension for the seeded enantiomers (i.e. L-threonine) can be neglected. Analysing this and other experiments, $\mathrm{k}_{\mathrm{b}}$ could be determined by matching experimental and theoretical profiles. First results for the estimated parameters describing growth and nucleation rate of the seeded enantiomer are summarised in Eqs. (24-25).

L-threonine (seeded):

Crystal growth rate: $\mathrm{G}^{(1)}=\mathrm{k}_{\mathrm{g}}^{(1)} \cdot\left(\mathrm{S}_{(1)}-1\right)=2.5 \cdot 10^{-7} \mathrm{~m} / \mathrm{s} \cdot\left(\mathrm{S}_{(1)}-1\right)$

Nucleation rate: $\quad \mathrm{B}^{(1)}=\mathrm{k}_{\mathrm{b}}^{(1)} \cdot\left(\mathrm{S}_{(1)}-1\right)^{2}=6.6 \cdot 10^{3} \mathrm{~s}^{-1} \cdot\left(\mathrm{S}_{(1)}-1\right)^{2}$.

The profile is satisfactorily described by the model selected and for the established parameters for the crystal growth rate of L-threonine within the considered time period. With rising 
time (> $300 \mathrm{~min}$ ) these calculations show, of course, a disagreement compared to the observation, because the occurrence of the undesired counter-enantiomer has been neglectted in this first approximation. A better quantitative description of the results over the whole period of time up to the equilibrium state requires a more accurate model than provided by Eqs. (24) and (25) considering likewise the nucleation of the counter-enantiomer. However, it shall be reemphasised that for the description of the cyclic operation mode the consideration of this time period in which the desired enantiomer is preferentially crystallising is sufficient.

Additionally, the distribution function for L-threonine crystals was calculated based on the used model in order to examine its evolution. The mass-based distribution function $F_{m}$ is plotted against the particle size and the time in Figure 11. As expected, with rising time the mean value of the particle size is increasing. Beyond, it is obvious that the nucleation starts nearly at the beginning of the process and that the formed nuclei are also growing with time. Furthermore, Figure 12 reveals the experimentally determined as well as the theoretically predicted number-based distribution functions $F_{N}$ for different times. A satisfactory agreement is achieved between the experimental and theoretical values for the growth of the seed crystals.

\subsection{Studies on the cyclic operation mode}

The results performed in the cyclic operation mode (compare Figure 6) are shown in Figure 13 for two subsequent separation cycles with racemic composition at the beginning of the first cycle $\left(e_{0}=0\right)$ and a subcooling of $\Delta T=10 \mathrm{~K}$. The other experimental conditions can be found in Tab. 2 (run II). After addition of L-threonine seed crystals, the crystallisation process started and L-threonine crystallised (first branch). For the interruption of the crystallisation in this half cycle a "stop" rotation angle was chosen corresponding to an enantiomeric excess of $2 \%$ concerning the counter-enantiomer. In practice the process was interrupted earlier, since the resolution still proceeded during filtering and harvesting the crystallised enantiomer. For this reason it has been emerged that an exact time $t^{*}$ (Eq. 26) for ending the process is rather difficult to realise. However, as can be seen in Figure 13 the preferential 
crystallisation in a cyclic operation mode is feasible on principle.

For a quantification of this process and a possible comparison with other crystalliser configurations a new quantity, a so-called "productivity", has been introduced as it is defined in Eq. (26). Following the productivity of reactors in chemical engineering this quantity describes the mass of gained product (i.e. less mass of the enantiomer which is invested at the beginning in each half cycle for seeding as well as for generation of an initial excess in the first cycle) per unit time, at which the process is stopped, and per used mass of racemate.

$$
\text { Productivity: } \quad \operatorname{Pr}_{\mathrm{m}}^{(\mathrm{k})}\left(\mathrm{t}^{\star}\right)=\frac{\mathrm{m}_{\text {solid }}^{(\mathrm{k})}\left(\mathrm{t}^{\star}\right)-\mathrm{m}_{\text {seeds }}^{(\mathrm{k})}-\mathrm{m}_{\mathrm{excess}, 0}^{(\mathrm{k})}}{\mathrm{t}^{\star} \cdot 0.5 \cdot \mathrm{m}_{\text {racemate }}}, \mathrm{k}=\mathrm{L}-\text {-, D-threonine }
$$

It must be annotated that the inherent dead time for batch processes has been neglected in this first approach. In Tab. 3 the experimentally ascertained productivities $\operatorname{Pr}_{\mathrm{m}}$ are summarised for several separation sequences. Calculations based on the model described above predict a theoretical productivity $\operatorname{Pr}_{\mathrm{m}}$, theo in the range of $5.0 \cdot 10^{-6} \mathrm{~s}^{-1}$ and $1.2 \cdot 10^{-5} \mathrm{~s}^{-1}$. Both, experimental and theoretical productivities, are of the same order, although the measured values are slightly lower. Obviously, the filtration of the crystallised leads enforcedly to losses of small amounts of the solid product which result in the slight lower values. In detail, regarding the slopes of the polarimetric signal it has to be remarked that the crystallisation rate of $\mathrm{D}$-threonine is obviously higher than that of L-threonine. The shorter crystallisation time $t^{*}$ needed to achieve the given value for the "stop" rotation angle in case of D-threonine leads consequently to a higher productivity. Despite the use of the same sieve fractions slight differences in the surface area distributions of both enantiomers could be observed which might be the reason for the different slopes of the polarimetric signals.

Besides, the higher crystallisation rate for L-threonine in the second cycle in comparison to the first cycle started from racemic solution confirms the accelerating effect of an initial enantiomeric excess related to a higher driving force. In spite of some differences between simulated and measured profiles shown in Figure 13, the simplified model used enables to predict the trend of the optical rotation angle even for this cyclic operation mode performed under slightly different conditions. 


\section{Conclusions}

In this work, a quantification of the preferential crystallisation process was attempted based on experimental data for the test system L-/D-threonine/water. Main emphasis was placed on the description of the crystallisation in a time period in which only crystals of one enantiomer can be harvested and the counter-enantiomer does not occur.

The on-line polarimetry, in combination with density measurements as well as off-line surface area measurements of solid samples by microscopic investigation, was used for the estimation of crystal growth kinetics based on the method of moments. A first approach was found to give a satisfactory description of the measured data in the time region of concern.

Nevertheless, due to the inherent inaccuracies an experimentally based prediction of the nucleation rate seems to be difficult and leads to uncertain values. Therefore, the parameters for the nucleation rate were provided by estimation using experimental data as well as a rather simple model including both, crystal growth and nucleation for the exclusively crystallised enantiomer. Model simulations and measurements show a first satisfying agreement, even though more experiments at different crystallisation temperatures, with seed crystals of different sizes etc. are necessary to receive a more generally valid model.

Preliminary experiments show that the preferential crystallisation in the cyclic operation mode is feasible and that the obtained productivity is comparable with theoretical predictions applying the considered model. On the basis of the developed model which will be further refined, different crystalliser configurations and controlling concepts are currently investigated. 


\section{Notation}

Latin symbols

$\begin{array}{lll}a_{i} & \text { surface area of a single crystal } & \\ A & \text { surface area } & \mathrm{m}^{2} \\ B & \text { nucleation rate } & \mathrm{s}^{-1} \\ D & \text { width of crystals } & \mathrm{m} \\ D_{0} & \text { density of pure solvent } & \mathrm{kg} / \mathrm{m}^{3} \\ e e & \text { enantiomeric excess } & - \\ F_{A} & \text { surface area distribution function } & \mathrm{m}^{-2} \\ F_{m} & \text { mass distribution function } & \mathrm{kg} / \mathrm{m} \\ F_{N} & \text { number distribution function } & \mathrm{m}^{-1} \\ F_{n u c} & \text { number distribution function of nuclei } & \mathrm{m}^{-1} \\ g & \text { exponent for growth rate } & - \\ G & \text { (global) crystal growth rate } & \mathrm{m} \cdot \mathrm{s}^{-1} \\ k_{d} & \text { calibration constant of the density meter } & \mathrm{kg} / \mathrm{m}^{3} \\ k_{p o l} & \text { calibration constant of the polarimeter } & \circ \\ k_{V} & \text { volume shape factor } & - \\ I & \text { length of cuvette } & \mathrm{m} \\ L & \text { crystal length } & \mathrm{m} \\ m & \text { mass } & \mathrm{kg} \\ N & \text { number of particles } & - \\ P r & \text { productivity } & \mathrm{s}^{-1} \\ S & \text { supersaturation degree } & - \\ t & \text { time } & \mathrm{s} \\ t^{*} & \text { stop time for cyclic operation mode } & \mathrm{s} \\ T & \text { temperature } & { }^{\circ} \mathrm{C}, \mathrm{K} \\ V & \text { volume } & \mathrm{m} \\ W_{(k)} & \text { weigth fraction (mass of component } \mathrm{k} \mathrm{per} \mathrm{total} \mathrm{mass)} & \mathrm{kg} / \mathrm{kg} \\ x & \text { effective particle diameter } & \mathrm{m} \\ & \text { m } & \end{array}$

Greek symbols

$\begin{array}{lll}\alpha & \text { optical rotation angle } & \circ \\ \lambda & \text { wavelength }(589 \mathrm{~nm}) & \mathrm{nm} \\ \mu_{j} & \text { j-th moment } & \mathrm{m}^{\mathrm{j}} \\ \rho & \text { density } & \mathrm{kg} / \mathrm{m}^{3} \\ \rho_{0} & \text { density of pure solvent } & \mathrm{kg} / \mathrm{m}^{3}\end{array}$

Subscripts and superscripts

$\begin{array}{ll}\text { C } & \text { crystal } \\ \text { cryst } & \text { crystallisation, crystalliser } \\ d & \text { density } \\ \text { eq } & \text { equilibrium }\end{array}$




$\begin{array}{ll}\text { exp } & \text { experimental } \\ k & \text { number specifying the enantiomer }(1=\text { L-thr, } 2=\text { D-thr }) \\ j & \text { number for the } \mathrm{j} \text {-th moment } \\ L & \text { length } \\ \text { liquid } & \text { liquid phase } \\ m & \text { mass, mixture } \\ N & \text { number } \\ \text { nuc } & \text { nucleation } \\ \text { pol } & \text { polarimeter } \\ S & \text { solid phase } \\ \text { sol } & \text { solution } \\ \text { theo } & \text { theoretical } \\ \text { tot } & \text { total } \\ V & \text { volume } \\ W & \text { water } \\ O & \text { initial }\end{array}$

\section{Acknowledgements}

The authors gratefully acknowledge the discussions with Heike Lorenz. Thanks are also due to Daniel Polenske for performing a multitude of experimental results.

\section{References}

[1] Collins, A.N., Sheldrake, G.N., Crosby, J. (1994), Chirality in Industry: The Commercial Manufacture and Applications of Optically Active Compounds, John Wiley \& Sons

[2] Collins, A.N., Sheldrake, G.N., Crosby, J. (1997), Chirality in Industry II: Developments in the Manufacture and Applications of Optically Active Compounds, John Wiley \& Sons

[3] Jacques, J., Collet, A., Wilen, S.H. (1994), Enantiomers, racemates and resolutions, Malabar: Krieger

[4] Sheldon, R.A., Hulshof, L.A., Bruggink, A., Leusen, F.J.J., van der Haest, A.D., Wijnberg, H. (1990), Crystallization techniques for the industrial synthesis of pure enantiomers, Proceedings Chiral '90 Symposium Manchester, 101-107

[5] Collet, A. (1999), Separation and purification of enantiomers by crystallisation methods, 
Enantiomer 4, 157-172

[6] Beilles, S., Cardinael, P., Ndzié, E., Petit, S., Coquerel, G. (2001), Preferential Crystallisation and comparative crystal growth study between pure enantiomer and racemic mixture of a chiral molecule: 5-ethyl-5-methylhydantoin, Chem. Eng. Sci. 56, 2281-2294

[7] Courvoisier, L., Mignot, L., Petit, M.N., Sprendgard, U., Hedtman, U., Coquerel, G. (2002), Preferential crystallization of ( \pm )-5(4'-bromophenyl)-5-methylhydatoin. Comparison between SIPC and AS3PC processes at $2 \mathrm{I}$ and 10 / scales, Proceedings of the $9^{\text {th }}$ International Workshop on Industrial Crystallization BIWIC 2002, Halle-Wittenberg, September, $11^{\text {th }}-12^{\text {th }} 2002$

[8] Matsuoka, M., Hasegawa, H., Ohori, K. (1990), Purity Decrease of L-Threonine Crystals in Optical Resolution by Batch Preferential Crystallization, in: Crystallization as a Separation Process, American Chemical Society, 251-260

[9] Shiraiwa, T., Miyazaki, H., Kurokawa, H. (1994), Successive Optical Resolution by Replacing Crystallization of DL-Threonine, Chirality 6: 654-657

[10] Matsuoka, M. (1997), Purity Drop in Optical Resolution of Racemic Mixtures, in: Separation and Purification by Crystallization, American Chemical Society, 59-72

[11] Profir, V.M., Matsuoka, M. (2000), Processes and phenomena of purity decrease during the optical resolution of DL-threonine by preferential crystallization, Colloids and Surfaces A: Physicochemical and Engineering Aspects 164, 315-324

[12] Grabowski, E.J.J. (2004), Enantiopure Drug Synthesis: From Aldomet to Imipenem and Beyond, Proceedings Chirality 2004 ISCD 16 (16 ${ }^{\text {th }}$ International Symposium on Chirality) New York, 51

[13] Buhse, T. Kondepudi, D.K., Hoskins, B. (1999), Kinetics of Chiral Resolution in Stirred Crystallization of D/L-Glutamic Acid, Chirality 11: 343-348

[14] Ramkrishna, D. (2000), Population Balances: Theory and Applications to Particulate Systems in Engineering, $2^{\text {nd }}$ edition, Academic Press, A Harcourt Science and Technology Company

[15] Bogacki, P., Shampine, L. (1989), A 3(2) pair of Runge-Kutta formulas, Appl. Math. Letters 2, 1-9

[16] Garside, J., Gibilaro, L.G., Tavare, N.S. (1982), Evaluation of crystal growth kinetics from desupersaturation curve using initial derivatives, Chem. Eng. Sci. 37, 1625-1628

[17] Glade, H., Ilyaskarov, A.M., Ulrich, J. (2004), Determination of Crystal Growth Kinetics Using Ultrasonic Technique, Chem. Eng. Technol. 27 (4), 736-740 
[18] Mullin, J.W., Garside, J. (1967), The crystallization of aluminium potassium sulphate: a study in the assessment of crystallizer design data. Part I: Single crystal growth rates, Trans. Inst. Chem. Eng. 45, T2 85-90

[19] Hulburt, H.M., Katz, S. (1964), Some problems in particle technology: A statistical mechanical formulation, Chem. Eng. Sci. 19, 555-574

[20] Randolph, A.D., Larson, M.A. (1988), Theory of Particulate Processes: Analysis and Techniques of Continuous Crystallization, $2^{\text {nd }}$ edition, Academic Press, Inc. Harcourt Brace Jovanovich, Publishers

[21] Mersmann, A. (2001), Crystallization Technology Handbook, $2^{\text {nd }}$ edition, Marcel Dekker, Inc.

[22] Dandekar, D.V., Gaikar, V.G. (2003), Precipitation of Curcuminoids from Hydrotrope Solutions: Crystal Nucleation and Growth Kinetics from Batch Experiments, Separation Science and Technology 38 (15), 3625-3644

[23] Alvarez-Rodrigo, A., Lorenz, H., Seidel-Morgenstern, A. (2004), Online Monitoring of Preferential Crystallization of Enantiomers, Chirality 16: 499-508

[24] Elsner, M.P., Lorenz, H., Seidel-Morgenstern, A. (2003), Preferential crystallisation for enantioseparation - New experimental insights indispensable for a theoretical approach and an industrial application, Proceedings of the $10^{\text {th }}$ International Workshop on Industrial Crystallization BIWIC 2003, Rouen, September, $4^{\text {th }}-5^{\text {th }} 2003,18-25$

[25] Wulkow, M., Gerstlauer, A., Nieken, U. (2001), Modeling and simulation of crystallization processes using parsival, Chem. Eng. Sci. 56, 2575-2588

[26] Lorenz, H., Sapoundjiev, D., Seidel-Morgenstern, A. (2003), Solubility Equilibria in Chiral Systems and Their Importance for Enantioseparation, Eng. Life Sci. 3, 132-136

[27] Elsner, M.P., Lorenz, H., Seidel-Morgenstern, A. (2003), Enantioseparation using crystallisation by entrainment - some engineering aspects, AIChE Annual Meeting, San Francisco, September, $16^{\text {th }}-21^{\text {st }} 2003$ 


\section{Table captions}

Table 1: Summary of preliminary experiments performed in a batchwise set-up for preferential crystallisation in order to study effects of stirrer speed, seed amount, surface area of seeds, initial enantiomeric excess, extent of subcooling and crystallisation temperature (see also $[23,24]$ ).

Table 2: Overview of two experiments devoted to study the growth rates and the cyclic behaviour.

Table 3: Experimentally and theoretically determined productivities for the cyclic operation mode (theoretically predicted values are in brackets).

\section{Figure captions}

Fig. 1: Principle of preferential crystallisation illustrated in ternary phase diagram.

Fig. 2: Typical polarimetric signal providing qualitative information about the process of crystallisation by entrainment.

Fig. 3: Concept of the preferential crystallisation process and a possible operation mode based on two consecutive half cycles.

Fig. 4: Physical interpretation for the model used of the preferential crystallisation process.

Fig. 5: Illustration of typical shapes of the investigated crystals of threonine.

Fig. 6: Experimental set-up for the cyclic operation mode (compare Figure 3).

Fig. 7: Typical optical rotation angle curves (left) and trajectories (right) for reproducible, complete batch experiments until the equilibrium state is established (cf. run I, Tab. 2).

Fig. 8: Distribution of the particle surface area for an analysed basic population of $N=500$ seeds. 
Fig. 9: The temporal increase of the total surface area and the second moment, respectively as well as a desupersaturation curve (left). The evolution of the experimentally determined total surface area was fitted with an empirical function as shown in the left diagram. Plot of crystal growth vs. supersaturation degree exemplified for one experiment (cf. run I in Tab. 2).

Fig. 10: Comparison of the experimental $\alpha$-curve obtained by seeding with L-threonine crystals with the $\alpha$-curve simulated according to the ascertained crystallisation parameters for crystal growth and different values for the nucleation rate constant $k_{b}$ (indicated is the range used for parameter estimation and which is also of concern for the cyclic operation mode). Experimental conditions cf. run I, Tab. 2.

Fig. 11: Evolution of the particle size distribution under typical conditions mentioned above (compare Eqs. 1-4). For the sake of clarity the axis for the mass density function is enlarged in the right-hand diagram.

Fig. 12: Comparison between experimentally determined and theoretically predicted distribution functions for several times. Model parameters cf. Eqs. (24-25), Experimental conditions cf. run I, Tab. 2.

Fig. 13: Comparison of the experimental polarimetric signal for two subsequent separation cycles with the $\alpha$-curves simulated according to the ascertained crystallisation parameters $\left(k_{g}=2.5 \cdot 10^{-7} \mathrm{~m} / \mathrm{s}, \mathrm{k}_{\mathrm{b}}=6.6 \cdot 10^{3} \mathrm{~s}^{-1}\right)$. Seeding with L-threonine and $\mathrm{D}$ threonine, respectively is marked by arrows. 